Schmerz 2009 $23: 337-338$

DOI 10.1007/s00482-009-0832-3

(c) Deutsche Gesellschaft zum Studium des Schmerzes. Published by Springer

Medizin Verlag - all rights reserved 2009

\author{
L. Radbruch $\cdot$ F. Nauck ${ }^{2}$ \\ ${ }^{1}$ Klinik für Palliativmedizin, RWTH Aachen \\ ${ }^{2}$ Georg-August-Universität Göttingen
}

\title{
Forschung oder Klinik
}

\section{Klinische Forschung}

In der Erforschung von chronischen Schmerzen haben deutsche Arbeitsgruppen aus dem Bereich der Grundlagenwissenschaften international einen hohen Ruf erreicht. Die klinische Schmerzforschung ist dagegen im Vergleich weniger eminent. Gerade dieser Bereich, die Anwendung von neuen Konzepten und Interventionen am Patienten, ist aber von hoher Bedeutung, wenn Forschungsergebnisse den Patienten zugute kommen sollen.

„Der Schmerz" ist sehr an der klinischen Forschung und an der praktischen Anwendung von Forschungsergebnissen im klinischen Alltag interessiert. In der vorliegenden Ausgabe finden Sie Beiträge zu Akupunktur, zum Schmerzempfinden und zu neuropathischen Schmerzen. Von den Originalarbeiten ist allerdings nur die Arbeit zum Einfluss von Akupunktur auf postoperative Schmerzen, Übelkeit und Erbrechen nach viszeralen Eingriffen als randomisierte kontrollierte Studie angelegt [3], die anderen Originalarbeiten stellen epidemiologische Auswertungen dar.

Braucht Deutschland also mehr klinische Forschung? Dazu sind Förderschwerpunkte notwendig, wie sie z. B. in den Netzwerken zu Kopfschmerz/Migräne, Rückenschmerzen und neuropathischen Schmerzen umgesetzt sind, die seit 2002 vom Bundesministerium für Bildung und Forschung gefördert werden. Beiträge aus diesen Netzwerken werden in „Der Schmerz" veröffentlicht [1, 2, 4].

Vielleicht fehlen also eher die klinischen Forscher, die ihr Interesse an Forschung mit dem Interesse an der klinischen Tätigkeit am Patienten verbinden. Es ist in Deutschland sicher nicht die Regel, dass eine solche Kombination geför- dert wird. In vielen Einrichtungen, auch in den Universitäten, wird Forschung noch als Gegensatz zur klinischen Versorgung gesehen oder bestenfalls als Hobby für die Zeit nach Dienstende, nachdem die Patienten versorgt sind. Wir brauchen in Deutschland bessere Bedingungen im klinischen Alltag für Forschungstätigkeiten, z. B. durch zusätzliche Forschungsstellen in den Schmerzambulanzen und -kliniken.

Vor allem aber brauchen wir Persönlichkeiten, die das Interesse an Forschung mit dem Interesse an der klinischen Tätigkeit am Patienten verbinden. Nur so können wir sicherstellen, dass die Forschungsfragen für die klinische Praxis relevant sind, und dass die Ergebnisse der Forschung in der klinischen Praxis auch umgesetzt werden können.

Einer der bedeutenden Schmerztherapeuten in Deutschland, der diese Eigenschaften kombinierte, ist vor Kurzem gestorben. Michael Strumpf hat seine schmerztherapeutische Ausbildung in der Klinik für Anästhesiologie, Intensiv-, Palliativ- und Schmerzmedizin am Berufsgenossenschaftlichen Universitätsklinikum Bergmannsheil in Bochum begonnen, in der Arbeitsgruppe von Michael Zenz, und wurde dort schon früh mit der klinischen Forschung konfrontiert. Seine Publikationen in „Der Schmerz“ zeigen das weite Spektrum der klinischen Forschungen. In einer epidemiologischen Untersuchung der Opioidverordnungen wies er die Unterversorgung in der Region nach, denn nur 10\% der Ärzte verordneten Opioide, und nur wenige Patienten erhielten eine Opioidtherapie über einen längeren Zeitraum [8]. Untersuchungen zu neuen
Applikationsformen in der Opioidtherapie zeigten die vergleichbare Wirksamkeit z. B. von einer Morphinlösung mit retardierter Galenik [5]. Einen Schwerpunkt seiner Forschung stellten die Untersuchungen zur kognitiven Leistungsfähigkeit von Schmerzpatienten unter chronischer Opioidtherapie dar, die ebenfalls in „Der Schmerz“ veröffentlicht wurden $[6,7]$. Diese Untersuchungen zeigten, dass eine Langzeitopioidtherapie nicht zu einer Einschränkung der verkehrsrelevanten Leistungen führen muss, und waren damit eine wesentliche Voraussetzung für die geplante Änderung der Straßenverkehrsordnung, die nun das Führen eines Kraftfahrzeuges zulässt, wenn eine Opioidtherapie sachgerecht für die Therapie chronischer Schmerzen verordnet wurde.

Michael Strumpfs persönlicher Werdegang führte ihn weg von der Forschung. Als Chefarzt einer großen anästhesiologischen und schmerztherapeutischen $\mathrm{Ab}$ teilung in Bremen blieb keine Zeit für klinische Studien. Jedoch blieb der Drang nach der Verbindung von Forschung und Patientenversorgung, der schließlich zu seiner Bewerbung auf die Universitätsprofessur für klinische und experimentelle Schmerztherapie an der Universität Göttingen führte. Im Juli 2008 wurde er auf diese Professur berufen. Seine Pläne für die klinische Schmerzforschung wurden durch seinen frühen Tod jäh unterbrochen.

Michael Strumpf verkörperte das $\mathrm{Zu}$ sammenspiel von Klinik und Forschung und hat beispielhaft die patientennahe Forschung umgesetzt. Er behielt dabei stets im Auge, dass Forschung kein Zweck an sich ist, sondern mit den neu 
gewonnenen Erkenntnissen den betroffenen Patienten helfen soll. Mit der klinischen Forschung können die anfallenden Fragen aus der klinischen Praxis beantwortet werden, sie stellt damit eine notwendige Ergänzung zur Grundlagenforschung dar.

Michael Strumpf gehörte mit uns zu einer neuen Generation von Schmerztherapeuten, wir haben uns über unsere Projekte in verwandten Forschungsgebieten ausgetauscht und gemeinsame Projekte und Publikationen durchgeführt. Wir werden ihn bei der weiteren Entwicklung der Schmerztherapie in Deutschland vermissen.

\section{Korrespondenzadresse \\ Prof. Dr. L. Radbruch}

Klinik für Palliativmedizin, RWTH Aachen

Pauwelssstr. 30, 52074 Aachen

LRadbruch@ukaachen.de

\section{Literatur}

1. Baron R, Tölle TR (2009) Schmerz und QST. „Messen, was messbar ist". Schmerz 23(1):5-6

2. Geber C, Scherens A, Pfau D et al (2009) Zertifizierungsrichtlinien für QST-Labore. Schmerz 23(1):65-69

3. Grube T, Uhlemann C, Weiss T, Meissner W (2009) Einfluss von Akupunktur auf postoperative Schmerzen, Übelkeit und Erbrechen nach viszeralen Eingriffen: Eine prospektive, randomisierte Vergleichsstudie mit Metamizol und Standardbehandlung. Schmerz 23:360-369

4. Limmroth V, Hubrecht L, Diener HC (2004) Schneller, höher, weiter. Aktuelles zur akuten und prophylaktischen Behandlung der Migräne. Schmerz 18(5):398-403

5. Strumpf M, Donner B, Zenz M (1995) Morphin-Retardgranulat zur Therapie inkurabler Schmerzen tumorbedingter und nichttumorbedingter Genese. Pilotstudie. Schmerz 9(3):140-146

6. Strumpf M, Köhler A, Zenz M (1997) Opioide und Fahrtüchtigkeit. Schmerz 11(4):233-240

7. Strumpf M, Willweber-Strumpf A, Herberg KW, Zenz M (2005) Sicherheitsrelevante Leistungsfähigkeit von Schmerzpatienten unter Langzeitopioidtherapie. Schmerz 19(5):426-433

8. Willweber-Strumpf A, Zenz M, Strumpf M (1992) Verschreibung von Betäubungsmitteln: Analyse der ambulanten Versorgung bei Patienten der AOK. Schmerz 6(4):255-259

\section{H. Kirchner, W. Kirchner Professionelles Management im Krankenhaus}

Erste Hilfe für leitende Ärztinnen und Ärzte

Thieme-Verlag 2009, 335 S., 106 Abb., 59 Tab., (ISBN 978-3-13-148111-5), 149.00 EUR

Offen gestanden wirkt der Titel diess

Buches irritierend: Seit wann wurden Krankenhäuser denn von Amateuren geführt? Der herablassend klingende zweite Teil des Titels wird dem Inhalt auch nicht gerecht. Damit es aber nicht gleich zu negativ klingt: Es handelt sich hier wirklich um ein sehr nützliches Buch, das von kompetenten Autoren verfasst wurde.

Das Buch macht Mediziner vertraut mit den gegenwärtig angewendeten Managementtechniken, die aus der Industrie kommen und zunehmend auch im Krankenhausbereich Anwendung finden. Die Ärzte haben sich diesen Strategien anzupassen. Während früher der Arzt maßgeblich in der Führung eines Krankenhauses war, haben jetzt überwiegend Betriebswirte Führungs- und Entscheidungsbefugnis. Ob diese Techniken wirklich auf Dauer so erfolgreich sind, ist zweifelhaft. Schließlich gab es noch nie so viele Unternehmenspleiten wie in jüngerer Zeit (auch vor der Finanzkrise!).

Es würde den Rahmen der Rezension sprengen, auf einzelne Punkte dieses Buches einzugehen. Jedenfalls sei jedem Facharzt geraten, sich allmählich mit der Thematik betriebswirtschaftlichen Managements im Praxis- und Krankenhausbereich zu befassen. Dafür liefert dieses Buch eine sehr gute Grundlage. Auch wenn Ärzten immer mehr Managementaufgaben aufgebürdet werden, kommt man nicht umhin, ,,auf diesem Zug mitzufahren“. Natürlich ist intensives Controlling Basis einer erfolgreichen Geschäftsführung, aber man kann sich des Eindrucks nicht erwehren, dass manchmal übertrieben wird und geradezu "Kennzahlenfriedhöfe" angelegt werden. Steuerungsgrößen sind nur sinnvoll, wenn damit auch Steuerung möglich ist. Krankheit ist kein Industrieprodukt und in manchen Bereichen nicht steuerbar. Unter Priorisierung des Profitgedankens werden - allgemein bekannt - in Deutschland z. B. viel mehr Herzkatheter- untersuchungen, Angioplastien, Computertomogramme und MR-Tomografien durchgeführt als in anderen Ländern. Es sei an ein Zitat von Howard Hughes erinnert: „Es versteht sich von selbst, das man nicht zugleich hohe Prinzipien und hohe Profite haben kann."

Die Arbeitsverdichtung im medizinischen Bereich nimmt kontinuierlich zu, während man gleichzeitig eine Expansion des Management-Bereiches zur Kenntnis nehmen muss (Arbeitsverdünnung?). Das Aufbürden immer neuer Management- und Organisationslasten auf Ärzte hat nicht zuletzt dazu geführt, dass junge Ärzte „mit den Füßen entscheidend" unser Land verlassen und damit dem Gesundheitssystem nach einer sehr teuren Ausbildung nicht mehr zur Verfügung stehen.

Sinnvoll ist auf jeden Fall, sich mit der Thematik zu befassen und auch interessant. Dazu kann das vorliegende Buch zweifellos dienen. Es ist gut gegliedert, gut strukturiert und enthält viele, sehr interessante Aspekte des Krankenhaus-Managements, mit denen man vertraut sein sollte. Erfreulich wäre allerdings, wenn vielleicht ein Buch mit dem Titel „Professionelle Medizin im Krankenhaus - Erste Hilfe für Krankenhaus-Manager" geschrieben würde.

S. von Sommoggy, Vogtareuth 